\section{FINANCIAMIENTO GLOBAL Y CENTROS COMERCIALES EN BUENOS AIRES: UN ESTUDIO DEL CASO IRSA ${ }^{1}$}

Ivana Socoloff ${ }^{2}$

\section{Resumen}

Los trabajos desde las ciencias sociales preocupados por el estudio de los centros comerciales han realizado diferentes aportes que presentan mayoritariamente una mirada crítica respecto al tipo de sociabilidad y urbanidad que generan estos espacios. También encontramos investigaciones que los presentan como expresiones de la globalización y de la fijación del capital financiero. Sin embargo, son escasos los trabajos que ubican el foco en explicar de qué modo se fija el capital, estudiando a los actores locales de la producción y el financiamiento. En este sentido, nos proponemos analizar el proceso de concentración económica que ha

\section{GLOBAL FUNDING AND SHOPPING CENTERS IN BUENOS AIRES: A STUDY OF IRSA $^{1}$}

IvanaSocoloff ${ }^{2}$

\section{Abstract}

At social science level, studies focused on shopping centers criticize the type of sociability and urbanity generated by these spaces. It is also possible to find studies that refer to them as a mere expression of globalization and fixed financial capital. However there is little research focused on explaining how capital is fixed through the analysis of local actors involved in production and funding activities. In this way, we propose to analyze the process of economic concentration which shopping centers have been subject to in Buenos Aires, most notably in 
sufrido este sector de los shopping centers en Buenos Aires, con especial foco en el caso de la empresa Inversiones y Representaciones SA (IRSA), principal propietaria de centros comerciales de la ciudad. El argumento que presentamos aquí es que la concentración del sector se explica por la mediación local ejercida por IRSA en tanto canal de inversiones para fondos globales con interés en la inversión inmobiliaria en mercados emergentes.

\section{PALABRAS CLAVE: CENTROS COMERCIALES; CONCENTRACIÓN ECONÓMICA; FIJACIÓN URBANA DEL CAPITAL FINANCIERO; IRSA}

Fecha de recepción: 30-09-2013

Fecha de aceptación: 29-08-2014

1 El presente trabajo sintetiza y amplía algunos aspectos de la investigación que condujo a la realización de una tesis doctoral financiada por el Consejo Nacional de Ciencia y Tecnología (CONICET) de Argentina.

2 Argentina. Licenciada en Sociologia (UBA) con una Maestría en Ciencias Sociales en la EHESS (Paris) y un doctorado en la Facultad de Ciencias Sociales (UBA). Becaria postoctoral CONICET con sede en el Instituto de América Latina y el Caribe de la Facultad de Ciencias Sociales, Universidad de Buenos Aires. Correo electrónico: ivisoc@gmail.com the case of Inversiones y Representaciones SA (IRSA) - the main owner of shopping centers in Buenos Aires. This paper discusses the fact that the concentration experienced by this sector is the result of the local mediation pursued by IRSA as an investment manager of global funds targeting the real estate market in emergent economies.

\section{KEYWORDS: SHOPPING CENTERS; ECONOMIC CONCENTRATION; ALLOCATION OF FUNDS AT URBAN LEVEL; IRSA}

Received: 30-09-2013

Accepted: 29-08-2014

1 This paper summarizes and expands upon some aspects of a $\mathrm{PhD}$. dissertation funded by the National Council on Science and Technology (CONICET), Argentina.

2 Argentina. BA in Sociology, UBA. MSc in Social Sciences, EHESS (Paris). PhD in Social Sciences, Faculty of Social Sciences at UBA. CONICET Postdoctoral fellow at the Institute of Latin American and Caribbean Studies (IEALC) (Faculty of Social Sciences, University of Buenos Aires).Email: ivisoc@gmail.com 


\section{Introducción}

Los trabajos desde las ciencias sociales preocupados por el estudio de los centros comerciales ${ }^{3}$ se han focalizado en realizar aportes críticos respecto al tipo de sociabilidad y urbanidad que generan estos espacios. Diversos estudios provenientes de la antropología urbana, la sociología urbana o la geografía social, han calificado a los centros comerciales como "ciudades artificiales acondicionadas para el consumo" o aún "no-lugares" Trabajos más recientes, sin embargo, han discutido algunas de las representaciones existentes entre los investigadores ${ }^{6}$.

En Argentina y en otros países de América Latina las investigaciones sobre los centros comerciales también han descripto críticamente a los malls como "artefactos de la globalización"7, como "postales de la modernización"8, como emergentes del "retiro del Estado" y "la decadencia del espacio público". Para el caso de Buenos Aires en

3 Utilizamos indistintamente los términos centros comerciales, malls, shopping centers y shoppings.

4 Amendola, 2000

5 Augé, 1998.

6 Se ha sostenido, por ejemplo, que el shopping no constituiría una "isla", al mismo tiempo que cabría problematizar la "fractura" supuestamente existente entre el mall y su entorno. Véase por ejemplo Centner, 2012 para el caso de Buenos Aires o Pérez, Salcedo y Cáceres, 2012 para el caso de Santiago.

7 Mattos, 1999; Ciccolella, 1999.

8 Gorelik, 2004 [1997], p. 8.

9 Caprón, 2000. particular, los trabajos han seguido frecuentemente la trayectoria crítica que hemos planteado. Caprón, por ejemplo, ha observado la existencia de una escisión social entre el adentro y el afuera del shopping ${ }^{10}$ o han sido pensados como "lo otro" de la ciudad $^{11}$. Similares conclusiones han obtenido los estudiosos de centros comerciales que adoptaron una escala microsocial, quienes destacaron el mall como expresión de la producción de fragmentos urbanos $^{12}$ o de relaciones irritables [fractious] con el entorno ${ }^{13}$, o aun como creador de fronteras sociales y procesos de exclusión ${ }^{14}$.

Retomando los aportes de estos investigadores, entendemos que resulta necesario complementar estos estudios con otros nuevos que, superando la escala microsocial, permitan explicar cómo "la globalización" produce estos "artefactos" y contribuye a fragmentar el territorio. Para ello, resulta imprescindible desde nuestra perspectiva analizar a los actores locales que participan en el desarrollo de los malls, su administración y financiamiento. Es

\footnotetext{
10 Caprón, 1998 y 2000.

11 Rotbart, 2010.

12 Kozak (2008), por ejemplo, pone el énfasis en el centro comercial como parte de una producción de fragmentos urbanos, para lo cual da cuenta -entre otras cosas- de las fronteras físicas de acceso que separan al Abasto de su barrio.

13 Centner, 2012.

14 Carman, 2006.
} 
decir, aquellos que median entre el "capital global" y la ciudad para llevar adelante los emprendimiento.

Cabe destacar que estas preocupaciones se encuentran en línea con una serie de investigaciones recientes sobre los actores de la financiarización del sector inmobiliario ${ }^{15}$ interesadas en explicar las mediaciones entre las trasformaciones del capitalismo y los procesos locales. Si bien aún son escasos estos trabajos en América Latina, los recientes aportes de Cattaneo Pineda ${ }^{16}$ para el caso chileno, Sanfelici ${ }^{17}$ para el caso brasilero y David ${ }^{18}$ para el caso mexicano constituyen interesantes antecedentes sobre los que se apoya esta investigación.

En este caso, el objetivo aquí será explicar el proceso de concentración económica que ha sufrido este sector en la Ciudad Autónoma de Buenos Aires y el papel del capital global, con especial foco en el caso de la empresa Inversiones y Representaciones SA (en adelante, IRSA), principal propietaria de centros comerciales de la ciudad.

De modo tal que, por los límites de este trabajo, no será el objetivo de este texto dar cuenta del impacto más específico sobre el territorio en cada una de las inversiones (precios del suelo, impacto social, conflictos sociales, etc), tema que ha sido

15 Corpataux, Crevoisier y Theurillat, 2009; David y Halbert, 2010; Nappi-Choulet, 2009; Theurillat, 2011.

16 Cattaneo Pineda, 2011.

17 Sanfelici, 2013.

18 David, 2013.

154 revista invi № 84 / Agosto 2015 / Volumen No 30: 151-177 ampliamente abordado $\mathrm{n}$ investigaciones anteriores $^{19}$ y por los análisis de casos o micro análisis ${ }^{20}$. A su vez, por iguales razones, los efectos de estas inversiones sobre los financistas o sobre la organización del capital global no pueden sino ser abordados a partir de otra literatura general o macro análisis ${ }^{21}$.

El trabajo está organizado de la siguiente manera. En primer lugar, presentamos una breve aproximación al desarrollo y funcionamiento de los centros comerciales en Buenos Aires. En segundo lugar, describiremos las dinámicas actuales y su lugar dentro del sector de comercio minorista. A continuación, se explicará el proceso de expansión y concentración de este sector, hoy mayormente en manos de IRSA. Seguidamente, mostraremos cómo y por qué IRSA convirtió al negocio del shoppings en su principal sector en Argentina, a la vez que daremos cuenta del modo en que se financió este proceso. Con ello, nos proponemos aportar datos y elementos para explicar de qué manera el capital financiero global está produciendo Buenos Aires por medio de IRSA.

La metodología adoptada, de carácter cualitativo, se basa en el procesamiento y análisis de fuentes

19 Socoloff, 2013.

20 Remitimos a la bibliografía previamente citada sobre Buenos Aires.

21 Al respecto, véase por ejemplo Harvey, 2010 y 2011. 
secundarias difundidas por los actores que se procura analizar ${ }^{22}$. De modo tal que las fuentes son diversas y comprenden tanto documentos provenientes de ámbitos públicos (series estadísticas, documentos legislativos, estatales, financieros, etc), como archivos provenientes de empresas y cámaras empresarias o aún revistas y prensa especializada del sector ${ }^{23}$.

\section{La expansión de los centros de compras en Buenos Aires}

En este apartado desarrollaremos muy brevemente qué constituye un shopping en términos urbanos y económicos en el contexto argentino, a partir de las definiciones brindadas por agencias públicas y privadas.

Para arribar a una caracterización, es necesario tener en cuenta que los centros comerciales surgen en Argentina en un contexto diferente al estadounidense o europeo, caracterizados estos por la expansión de las clases medias y el auge económico ${ }^{24}$. Por el contrario, los shoppings argentinos aparecen en la década de los ' 80 y se consolidan a comienzos de

22 Sobre una explicación del carácter metodológico de este tipo de investigaciones, véase Halbert, 2013 y Socoloff, 2013.

23 Todas las fuentes seleccionadas para la confección de este artículo se citan en la bibliografía. Un listado mayor de fuentes sugeridas de consulta para este tipo de trabajos se encuentra en Socoloff, 2013

24 Caprón, 1997. los años '90, en un momento de crisis económica que castiga a los sectores medios, junto con un aumento del desempleo que alcanzó tasas del 17\% a mediados de la década. Otra característica es que su expansión suburbana se produjo sólo en los últimos años, destacándose mayormente por su localización en el centro de las ciudades, con gran accesibilidad mediante el transporte público ${ }^{25}$. Todo lo cual, también diferenció el fenómeno de los malls argentinos de sus pares brasileros o mexicanos.

Ahora bien, antes de avanzar en su caracterización, comencemos por aproximar una definición respecto a este objeto urbano. ¿De qué hablamos cuando hablamos de shopping centers en Buenos Aires? De acuerdo con la definición brindada por la Cámara Argentina de Shopping Centers, un shopping debe cumplir los siguientes requisitos: a) planificación y administración centralizadas; b) existencia de un fondo común para invertir en publicidad y promoción; c) oferta comercial variada en productos y servicios; d) única titularidad de explotación sobre la totalidad de las locales comerciales, admitiéndose como excepción la enajenación de los sectores denominados 'Tiendas Anclas'26.

25 Existen excepciones a este fenómeno que fueron los centros comerciales que actuaron como nodos en las redes de autopistas que se expandieron en los años '90. Ejemplos de ello, son el Unicenter, el Soleil Factory y el shopping Dot Baires.

26 Se entiende por "tiendas anclas" a los grandes locales destinados a atraer clientes, como podría ser un gran espacio de supermercado (http://www.casc.org.ar/, 2013).

revista invi № 84 / Agosto 2015 / Volumen № 30: 151-177 
Como se observa, el aspecto de la centralización de la administración resulta un elemento definitorio de este tipo de organizaciones, debiendo contar con un único propietario y una administración unifcada para todo el espacio, acercándolo al modelo norteamericano de centro comercial que se diferencia de, por ejemplo, el caso francés ${ }^{27}$.

Desde el punto de vista urbanístico, la normativa de la ciudad establece que un centro de compras es un "Complejo comercial con una superficie total no menor de veinticinco mil metros cuadrados (25.000m2) integrado como mínimo por: a) Un supermercado total o un supermercado más una supertienda; b) Un número de locales minorista y de prestación de servicios cuya suma de superficie sea por lo menos igual al total de la superficie requerida por los locales descriptos en el inciso a); c) Una playa para automóviles no menor a dos veces la superficie conjunta de los espacios destinados a la venta"28. En este sentido, la normativa procura diferenciar al centro comercial de espacios menores o "paseos de compras", que operan como galería comercial.

A pesar de algunos ejemplos que le anteceden, el uso de centro comercial fue admitido dentro del Código de Planeamiento de la Ciudad recién en 1993, es decir, tres años luego de creada la cámara de representantes empresariales que operó como

27 Caprón, 1997, 1999 y 2000.

28 Código de Planeamiento Urbano de la CABA, 2013. actor clave para lograr el reconocimiento ${ }^{29}$. Por su parte, no fue sino hasta 1997 que el Instituto Nacional de Estadística y Censos registró la actividad y comenzó a relevar datos sobre el comportamiento económico de los centros de compras. Hacia el año 2002, los documentos de la entidad afirmaban:

"En el transcurso de los últimos años, se han registrado grandes cambios en la actividad del comercio minorista, siendo uno de los más significativos la aparición de los Centros de compras ("Shopping Centers"). Se trata de un novedoso formato de comercio minorista que ofrece un muy extenso surtido de productos y servicios dentro de un espacio ambiental contenido en un diseño arquitectónico que proporciona comodidad y seguridad a los concurrentes. (...) El importante desarrollo alcanzado por los Centros de compras en nuestro país ha impulsado al INDEC a poner en marcha una encuesta de periodicidad mensual, destinada a conocer la evolución de las principales variables que describen su actividad." 30

Como se observa en la cita, la Institución reconoce el "novedoso formato de comercio minorista" que constituyen los centros de compras, caracterizados por su diseño arquitectónico y por el hecho de que los locales se alquilan y abonan un porcentaje de la facturación. Fue entonces durante la segunda mitad de la década de los '90 que los shoppings se instalaron como espacios de consumo entre la

\section{Caprón, 1997.}

30 INDEC, 2000, p.2. 
población de la ciudad. Por ello, los organismos estatales de estadística reconocieron la especificidad de este fenómeno, con la colaboración de las entidades empresariales que ofrecieron los datos de base ${ }^{31}$.

\section{Tendencias actuales en el consumo minorista en Buenos Aires}

En este apartado mostraremos y explicaremos el crecimiento y consolidación de los centros comerciales como ámbitos privilegiados del consumo minorista de la ciudad desde los años '90 a la actualidad. Este crecimiento en ventas da cuenta del atractivo de estas inversiones para agentes locales e internacionales. A pesar de lo cual, destacaremos que los shoppings compiten con otras formas de consumo minorista también expandidas en Buenos Aires, como son los llamados circuitos de

31 Cabe destacar que el INDEC encuesta a las unidades definidas por la Cámara Argentina de Shopping Centers como tales. outlets (comercios con ofertas), por un lado, y los mercados textiles informales, por el otro.

Un primer indicador de la expansión es su crecimiento numérico. Desde el año 1997 a la actualidad $^{32}$, resulta llamativa la casi duplicación del número de centros comerciales en el Gran Buenos Aires (en adelante, GBA) ${ }^{33}$ de 20 centros en enero de ese año a 36 shoppings en junio de 2013. De ellos, 18 se encuentran actualmente ubicados en Ciudad de Buenos Aires (en adelante, CABA) y los 18 restantes en el resto del GBA (ver figura 1). En Ciudad de Buenos Aires, 14 de los 18 centros de compras tienen menos de 100 locales y los 4 restantes, más de 100 locales, mientras que en los 24 partidos de la provincia de Buenos Aires relevados, 12 de los 18 centros de compras tienen menos de 100 locales y los 6 restantes, más de 100 locales.

La tipología de estos espacios es variada, pero todos ellos han logrado consolidarse presentándose como ámbitos de consumo "seguros" y "vigilados"34. Todo lo cual, ha atraído principalmente a adolescentes y a familias que se congregan en los patios

321997 es el año de inicio de la Encuesta de Shopping Centers diseñada por el INDEC.

33 Gran Buenos Aires: área comprendida por la CABA más los 24 partidos: Almirante Brown, Avellaneda, Berazategui, Esteban Echeverría, Ezeiza, Florencio Varela, General San Martín, Hurlingham, Ituzaingó, José C. Paz, La Matanza, Lanús, Lomas de Zamora, Malvinas Argentinas, Merlo, Moreno, Morón, Quilmes, San Fernando, San Isidro, San Miguel, Tigre, Tres de Febrero y Vicente López.

34 Al respecto, ver Caprón, 1998 y 2000; Rotbart, 2010. 
FIGURA 1: CANTIDAD DE SHOPPINGS SEGÚN LOCALIZACIÓN EN EL GBA

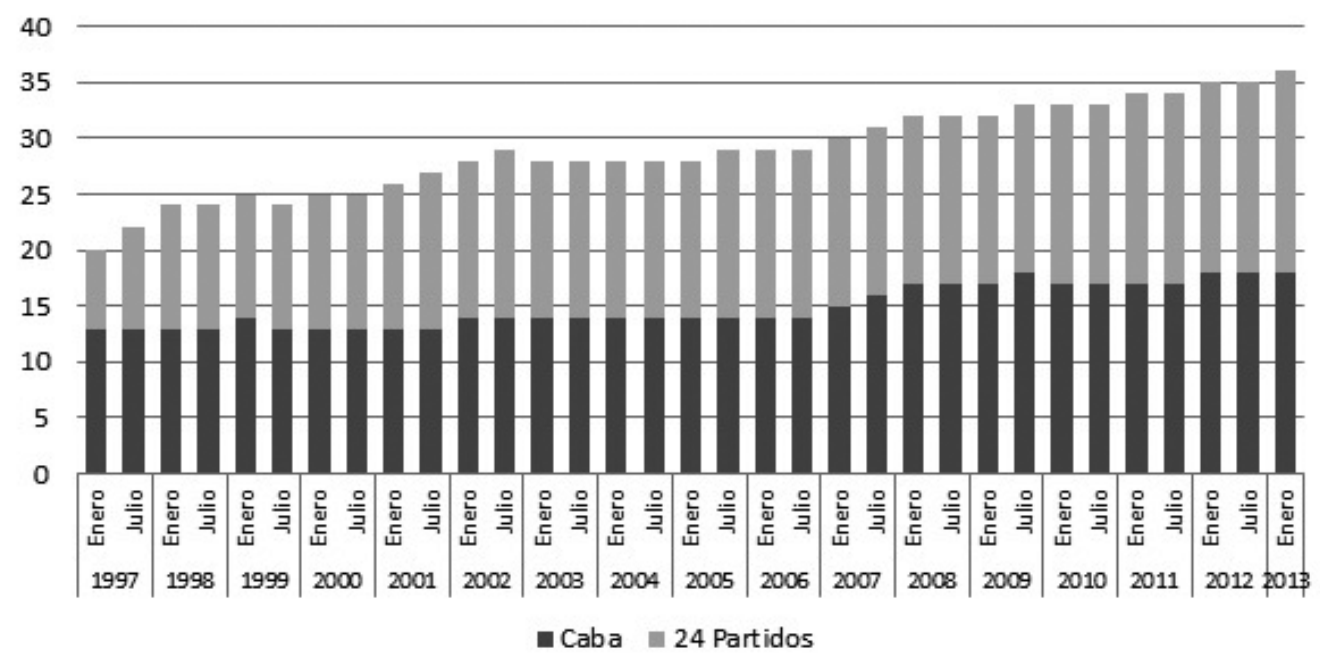

Fuente: Elaboración propia en base a datos de la "Encuesta de Centros Comerciales" -INDEC

FIGURA 2: VENTAS DE CENTROS DE COMPRAS. SERIES A PRECIOS CONSTANTES Y DESESTACIONALIZADA. BASE ABRIL 2008 $=100$

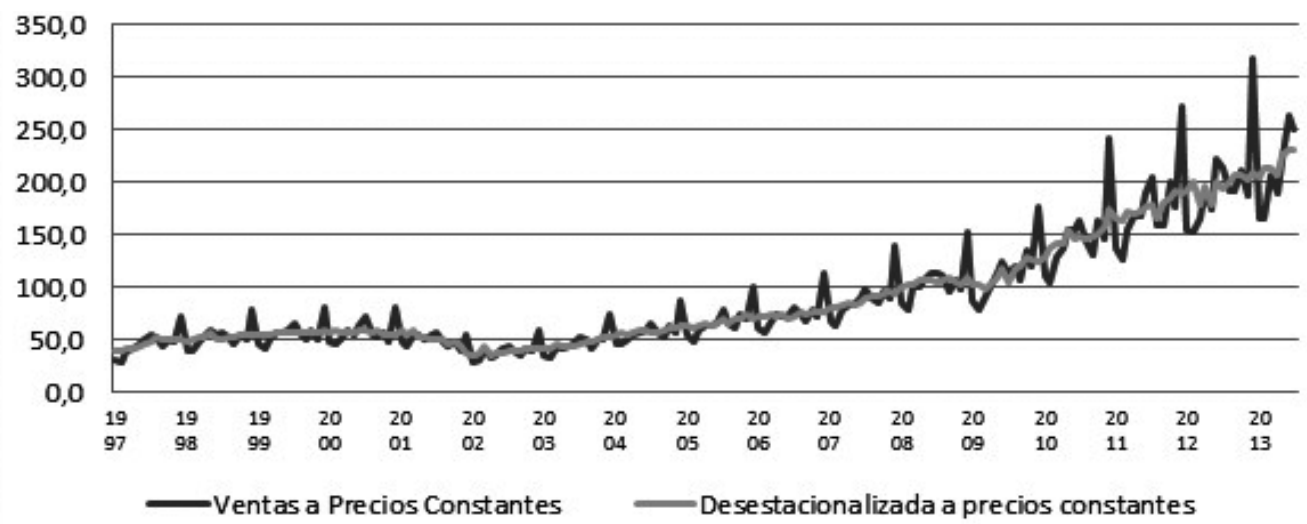

Fuente: Elaboración propia en base a datos de la "Encuesta de Centros Comerciales", INDEC 
de comidas, lugares de encuentro en torno a los cuales se organiza el centro comercial. En este sentido, y como destacan los estudios de mercado, los shoppings de Buenos Aires tienden a ser lugares de paseo, más que de compra y constituyen espacios de reproducción de la clase media ${ }^{35}$.

Otro indicador de la consolidación de los centros comerciales como forma de consumo minorista en Buenos Aires, es la información que surge sobre sus ventas. Desde 1997 a la actualidad, vemos una expansión clara de los niveles de ventas, como se puede apreciar en el Gráfico 2, construido a precios constantes.

Dicho crecimiento, propio de los últimos diez años, respondió en el caso de Buenos Aires a numerosas condiciones, que hemos desarrollado más ampliamente en otro trabajo, pero que sintetizaremos aquíi ${ }^{36}$. En primer lugar, cumplió un papel importante la creciente afluencia de turistas a la Argentina desde 2002 a la actualidad producto de la caída de los precios relativos argentinos, como se observa en el crecimiento de la tasa de ocupación de habitaciones hoteleras ${ }^{37}$. Asimismo, observamos la expansión de la capacidad de consumo local, por el crecimiento en el salario y el aumento

35 Caprón, 1997 y 1999.

36 Socoloff, 2013.

37 Este fenómeno se observa en todo el período, excepto en 2009 por la crisis mundial y la expansión de epidemia de la llamada "gripe A" o "gripe H1N1". del empleo, al menos entre 2003-200838; así como la disminución de los niveles de desempleo en relación al período pre-crisis.

Más recientemente, entre 2009 y 2012, el crecimiento de las ventas en los centros comerciales se explica por la inexistencia de instrumentos de ahorro a largo plazo y la inflación argentina que orientaron los ahorros al consumo minorista. Igualmente, tuvieron un papel importante la expansión del crédito al consumo de 33,8\% para los préstamos a las familias en pesos argentinos (entre noviembre de 2011 y noviembre de 2012), siendo el destino más destacado los préstamos con tarjeta, que se incrementaron en un $46,7 \%$ en ese período ${ }^{39}$.

Todo lo cual, es complementario al proceso de consolidación de esta forma de consumo entre distintos sectores sociales y al aún bajo nivel de penetración de los shoppings en el mercado argentino, en comparación con otros países de la región. En los últimos años también, los consultores especializados en el mercado inmobiliario destacan el atractivo que representa para los centros comerciales la existencia de promociones y descuentos con tarjetas de crédito para el consumo en días de la semana estipulados ${ }^{40}$.

38 Ver: Panigo y Neffa, 2009, p.14. Las series de datos analizados por los autores se extienden hasta 2009. Es probable que esta tendencia no se verifique para el período 2009-2012.

39 Según datos del Banco Central de la República Argentina (ver CEFID-AR, 2012, p. 3.)

40 L.J. Ramos, 2011, p. 4-12. 
Ahora bien, a pesar del crecimiento de los centros comerciales, es interesante notar que los circuitos de consumo en Buenos Aires se han transformado considerablemente en los últimos años ${ }^{41}$. Principalmente porque los consumidores han incorporado los locales de outlets y las ferias informales como ámbitos privilegiados. Por ello, los shoppings han debido adaptarse también, incorporándose recientemente a la oferta los llamados centros a "cielo abierto", como forma mixta entre un shopping tradicional y un paseo de compras al aire libre ${ }^{42}$.

Un caso paradigmático de las transformaciones en los circuitos de consumo en Buenos Aires lo constituye la imponente feria informal llamada "La Salada", ubicada en el partido de Lomas de Zamora (GBA). La misma concentra alrededor de treinta mil puestos de venta que funcionan dos veces por semana. En pequeños puestos de alrededor de $4 \mathrm{~m}^{2}$, se comercia principalmente indumentaria, mucha de la cual constituye réplicas ilegales de marcas famosas. Lo llamativo del caso es que, si bien no se cuenta con datos oficiales, se estima que

41 Ibid

42 Un ejemplo de lo cual lo constituye el shopping Arcos Gourmet en la CABA. las ventas en la Feria igualan o superan las ventas de los 36 shoppings del GBA ${ }^{43}$. De modo tal que, como se ve, los centros comerciales, a pesar de su creciente importancia, no dominan el mercado de comercio minorista en Buenos Aires.

\section{El proceso de concentración y financiamiento: el caso de APSA-IRSA}

Como mostraremos, el crecimiento y la expansión de las ventas en los centros comerciales fue correlativa con la concentración económica del sector en Buenos Aires, proceso al que nos referiremos en este apartado tomando como eje el caso de la empresa IRSA.

De los 18 shoppings de la CABA, IRSA controla en la actualidad seis de ellos, mientras que el Grupo de origen chileno Cencosud ${ }^{44}$ posee dos centros

43 No existen estimaciones oficiales. Las estimaciones realizadas por los propios representantes de la Feria ante la prensa, sostienen que las ventas allí superan las de los shoppings. Las difundidas por las consultoras afirman mientras que la facturación en los shoppings centers fue de $\$ 11.415$ millones en 2011 y en la Feria de la Salada de $\$ 11.520$ millones. Ver J.L.Ramos, 2011, p. 7.

44 EI CEO del grupo es el empresario de origen alemán radicado en Chile, Horst Paulmann. El Holding se extiende por varios países de América Latina incluyendo Brasil, Colombia y Perú además de Argentina y Chile. 
comerciales y el Grupo Village Cinemas SA ${ }^{45}$ posee participación en dos centros. Es decir, IRSA controla la tercera parte de los establecimientos, pero el 53\% del Área Bruta Locativa (ABL) y el 49\% en cantidad de locales (Ver Tabla 1). Y más importante aún, IRSA gestiona los tres shoppings más importantes de la ciudad en tamaño, tanto si consideramos el ABL (Paseo Alcorta, Abasto y DOT Baires) como según cantidad de locales comerciales (Abasto, DOT Baires y Alto Palermo). Expliquemos entonces cómo esta sociedad llegó a asumir una posición dominante.

La sociedad IRSA es hoy la principal propietaria de APSA (Alto Palermo Sociedad Anónima), que constituye la rama de la empresa abocada al negocio de los shoppings. Eduardo Elsztain es la cara visible de IRSA desde que tomó el control en 1991 de esta empresa "cáscara" que operaba en la Bolsa desde $1943^{46}$ y convirtió una compañía que daba pérdidas, en un grupo económico con activos

45 Controla el shopping Village Caballito y posee una participación en el Recoleta Mall. Village Cinemas S.A, es una sociedad argentina cuyos accionistas son Southern Screens Entertainment con el $70 \%$ del paquete accionario y Blue Ridge Investments, compañía inversora institucional de capitales estadounidenses. El principal socio de Southern es Summerfield K. Johnston Jr., quien fue director y CEO de Coca Cola.

46 Con empresa "cáscara" nos referimos a una empresa que, sin cumplir una finalidad específica, operaba en la Bolsa de Comercio de Buenos Aires habiendo cumplimentado todos los requisitos para ello. La estrategia consiste en dotar a la sociedad existente de un nuevo propósito, evitando así las gestiones para la creación de una nueva sociedad y su oferta pública. consolidados superiores a 6.600 millones de pesos argentinos. Con sólo algunos pocos trabajadores al comienzo, hoy emplea a más de 1500 personas que combinan el negocio inmobiliario con un conocimiento profundo del mercado de capitales ${ }^{47}$.

De la mano del magnate húngaro-estadounidense George Soros en sus comienzos, IRSA ingresó a los mercados mundiales llegando a cotizar sus acciones en la Bolsa de Nueva York (NYSE), y en NASDAQ a través de otras empresas del Grupo. La inversión en Argentina del fondo que lideraba Soros -el Quantum Fund- a comienzos de los años '90 se enmarcaba en el interés que estaban exhibiendo los mayores fondos internacionales en el sector inmobiliario de países periféricos ${ }^{48}$. En particular, el Quantum Fund mostró una temprana orientación hacia los mercados emergentes de América Latina en el contexto de la caída de la URSS y la crisis del este europeo, como lo relata el propio Soros ${ }^{49}$.

\section{David y Halbert, 2010}

49 Soros, 1995, p.63. 
TABLA 1: LISTADO DE PROPIETARIOS Y SU PARTICIPACIÓN DEL SEGMENTO DE LOS CENTROS COMERCIALES EN LA CIUDAD DE BUENOS AIRES

\begin{tabular}{|c|c|c|c|c|c|}
\hline Grupo propietario principal & Nombre de shopping & $\mathrm{ABL}$ & Locales $^{47}$ & $\% \mathrm{ABL}$ & $\%$ locales \\
\hline CENCOSUD & Factory Parque Brown & 31.468 & 91 & $8,88 \%$ & $6,20 \%$ \\
\hline CENCOSUD & Portal de Palermo & 32252 & 36 & $9,10 \%$ & $2,45 \%$ \\
\hline CINCUENTA Y UNO CERO OCHO S.A. & Caballito Shopping Center & 4611 & 52 & $1,30 \%$ & $3,54 \%$ \\
\hline DEL PARQUE SHOPPING CENTER SA & Del Parque Shopping & 5956 & 85 & $1,68 \%$ & $5,79 \%$ \\
\hline DENO SA & Devoto Shopping & 18741 & 93 & $5,29 \%$ & $6,34 \%$ \\
\hline FORTIN MAURE SA & El Solar de la Abadía & 6825 & 78 & $1,93 \%$ & $5,32 \%$ \\
\hline GALERÍA GENERAL GÜEMES S.A.I & Galería Güemes & 1820 & 24 & $0,51 \%$ & $1,64 \%$ \\
\hline GLA SA & Plaza Liniers & 5800 & 57 & $1,64 \%$ & $3,89 \%$ \\
\hline IRSA-APSA & Abasto de Buenos Aires & 41464 & 174 & $11,70 \%$ & $11,86 \%$ \\
\hline IRSA-APSA & Alto Palermo Shopping & 18701 & 143 & $5,28 \%$ & $9,75 \%$ \\
\hline IRSA-APSA & Paseo Alcorta & 52736 & 111 & $14,88 \%$ & $7,57 \%$ \\
\hline IRSA-APSA & Patio Bullrich & 11684 & 83 & $3,30 \%$ & $5,66 \%$ \\
\hline IRSA-APSA & Buenos Aires Design & 13769 & 62 & $3,88 \%$ & $4,23 \%$ \\
\hline IRSA-APSA & Dot Baires Shopping & 49527 & 152 & $13,97 \%$ & $10,36 \%$ \\
\hline SUTTON & Galerias Pacífico & 13991 & 128 & $3,95 \%$ & $8,73 \%$ \\
\hline VC1 - SRL (VILLAGE CINES + CARVAL de Cargill) & Recoletta Mall & 14894 & 75 & $4,20 \%$ & $5,11 \%$ \\
\hline VILLAGE CINES & Village Caballito & 5471 & 4 & $1,54 \%$ & $0,27 \%$ \\
\hline WALMART ARGENTINA S.R.L & Walmart & 24743 & 19 & $6,98 \%$ & $1,30 \%$ \\
\hline TOTAL & & 354453 & 1467 & $100 \%$ & $100,00 \%$ \\
\hline TOTAL PARTICIPACIÓN IRSA EN BUENOS AIRES & & & & $53,01 \%$ & $49,42 \%$ \\
\hline
\end{tabular}

Fuente: Elaboración propia en base a datos de la Cámara Argentina de Shopping Centers

47 Sin stands ni gastronómicos, según la CASC. 
Con un acceso casi irrestricto a los capitales internacionales y gracias a que el estado argentino se estaba deshaciendo de importantes inmuebles y tierras, IRSA se expandió durante la primera mitad de los ' $90^{50}$. Sin embargo, con las sucesivas crisis en América Latina durante la segunda mitad de la década (México y Brasil), los fondos de Soros comenzaron un proceso de desvinculación previo a la crisis argentina de 2001. Su salida desencadenó una reestructuración societaria, a partir de la cual CRESUD -otra empresa del grupo hasta entonces controladora de las propiedades rurales- se convirtió en su accionista principal. Un porcentaje mayoritario de las acciones de CRESUD, a su vez, quedaron en manos de la empresa "Inversiones Financieras del Sur S.A" (IFISA), una sociedad domiciliada en Uruguay, controlada en un 100\% -a su vez- por otra sociedad domiciliada en las Islas Bermudas (IFIS, "Inversores Financieros del Sur"), donde Elsztain se desempeña como presidente. En otras palabras, IRSA fue indirectamente adquirida por otras sociedades localizadas en paraísos fiscales, cuya composición accionaria en términos de origen de los capitales involucrados puede ser sólo objeto de rumores, dado que no es posible conocer su titularidad.

50 Socoloff, 2013.
Ahora bien, ¿cómo se convirtió esta pequeña empresa en un holding internacional en una década? Si repasamos sus comienzos, vemos que el actual CEO de la compañía orientó la nueva etapa de IRSA a la especialización de sus actividades, vinculadas a partir de 1991 sólo con el negocio inmobiliario. Dicha especialización, por la que han atravesado numerosas compañías en el mundo, actúa facilitando el análisis de los inversores internacionales que procuran firmas centradas en una sola actividad a la hora de calcular sus riesgos ${ }^{51}$.

En un comienzo, los desarrollos de IRSA se concentraron en el sector de vivienda, y en el reciclaje de oficinas y los comercios para renta ${ }^{52}$. Entre las áreas de intervención, se identificaban en 1992 cuatro segmentos sobre los que la Sociedad focalizaría sus actividades: "residencial", "comercial", "terrenos" e "inmuebles estatales", en ese orden. Dentro del sector residencial, IRSA se orientó en un primer momento al desarrollo de vivienda para la "clase media", pero rápidamente cambiará dirigiendo sus emprendimientos hacia los sectores medios altos y altos.

Otro sector considerado como estratégico para IRSA hacia 1992 era la adquisición de los

51 Halbert, 2013

52 En lo que los documentos empresariales llamaban "segmento comercial", se agrupaba a los comercios y oficinas destinadas al reciclaje y locación, cuya función principal sería la de producir un flujo de caja importante. 
denominados "terrenos estratégicamente ubicados", comprados para su posterior venta o desarrollo. La intermediación consistía en seleccionar "oportunidades" en parcelas, vendiéndolas luego a valores muy superiores de los originales. IRSA se proyectaba tempranamente como una empresa tenedora de tierras y, por lo tanto, proveedora de las constructoras. En este sentido, el momento de la compra fue siempre fundamental dentro de la estrategia de la compañía ${ }^{53}$, siendo sus principales y más importantes desarrollos producto de compras realizadas al Estado.

Las mismas fueron posibles, entre otras razones, por su acceso a los mercados de capitales internacionales, privilegio de pocas empresas argentinas a principios de los años '9054. La empresa recurrió a la estrategia de ampliar el capital incorporando nuevos socios o, más frecuentemente, a la emisión de obligaciones negociables a tasas norteamericanas de esa época (que rondaban el 4\% anual), produciendo tasas de rentabilidad en la región muy superiores, en dólares y con seguro de cambio ${ }^{55}$.

En otras palabras, desde el comienzo los empresarios de IRSA estuvieron convencidos de la importancia que tenía para el sector inmobiliario la

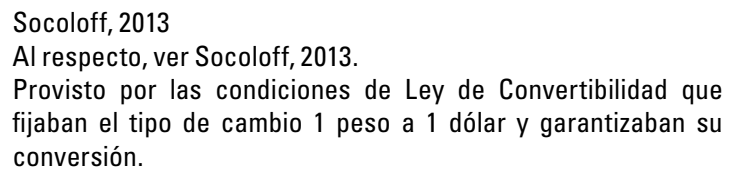

Provisto por las condiciones de Ley de Convertibilidad que fijaban el tipo de cambio 1 peso a 1 dólar y garantizaban su conversión.

transferencia de inmuebles y parcelas a manos privadas que realizaba el Estado en condiciones como las mencionadas. Como consta en los documentos empresariales, IRSA era homologada a los grandes grupos concentrados de la economía argentina de principios de los '90, partícipes del intenso proceso de privatizaciones. Sus empresarios entendían acertadamente que el acceso a capitales internacionales -con una tasa de interés en dólares internacional menor a la local, como decíamos- les otorgaba una ventaja comparativa respecto a sus competidores en el proceso de privatización de inmuebles estatales ${ }^{56}$.

En este contexto, la cotización en Bolsa resultaba un elemento único y distintivo cuyo monopolio mantuvo hasta recientemente, pues era el canal para obtener una participación accionaria en la empresa-vehiculo de inversiones locales y extranjeras, a la que aspiraba a convertirse IRSA. De esta manera, IRSA se transformará en un grupo especializado en captar recursos del sistema financiero y volcarlos al mercado inmobiliario.

Sin embargo, y luego de los efectos de la crisis mexicana de mitad de década, la situación de la empresa irá progresivamente cambiando. En este

\footnotetext{
56 Podían ofrecer sumas y condiciones de pago superadoras de la competencia en los procesos de remate de bienes públicos, como lo testimonian diversos funcionarios.
} 
sentido, un punto de inflexión dentro de la historia del holding lo constituye el año 1997. Es a partir de ese año que la compañía coloca en primer lugar de su estrategia a los inmuebles de renta. Entre dichos inmuebles, las oficinas "de categoría" resultan las más importantes, dado que aseguraban, en la situación posterior a la crisis del tequila, "inquilinos solventes". Por su parte, los shoppings, que en 1997 se ubicaban al final de la estrategia, se convertirán sin embargo en el principal negocio en los años subsiguientes ya que en sólo un año, IRSA llevará adelante un agresivo programa de compras de centros comerciales (tabla 2).

Aclaremos que, hasta 1997, IRSA era propietaria de acciones en solo tres sociedades ligadas al negocio de los shoppings centers, uno de las cuales estaba ubicado en Buenos Aires ${ }^{57}$. En este marco, y en sólo dos años, IRSA adquirió participaciones en sociedades controladoras de cinco centros comerciales de la ciudad de Buenos Aires, y de dos más

57 Sociedades en las que IRSA tenía participación: el Nuevo Norte Shopping Center en la provincia de Salta, una parte del Mendoza Plaza Shopping en la provincia de Mendoza y finalmente estaba SAMAP (Sociedad Anónima Mercado de Abasto Proveedor), que era la sociedad que se encontraba desarrollando el Mercado del Abasto, cuya compra IRSA había concretado en 1994 para desarrollo. fuera de ella ${ }^{58}$. Asimismo, y junto con la agresiva política de compra de shoppings, IRSA por medio de su subsidiaria Alto Palermo Sociedad Anónima (APSA) compró importantes reservas de tierras para el desarrollo de nuevos shoppings ${ }^{59}$. La mayor parte de estas sociedades fueron fusionadas bajo la órbita de SAMAP (Sociedad Anónima Mercado Abasto Proveedor), la titular del Centro Comercial "Abasto", que en 1998 cambió de nombre y se convirtió definitivamente en APSA, denominación que mantiene hasta la actualidad.

Para llevar adelante esta gran cantidad de adquisiciones ligadas al negocio específico de los shoppings, IRSA requirió de socios internacionales que aportaran capital. Dicho capital fue obtenido por medio de alianzas estratégicas con una empresa chilena y con un fondo ligado a Goldman Sachs. En términos societarios, APSA quedó bajo la órbita de "IRSA International Ltd" (la rama internacional de IRSA con sede en las Islas Vírgenes), conformándose hacia 1999 la participación en APSA de la siguiente manera:

58 Las Galerías Pacífico que luego vendió, los shoppings Paseo Alcorta, Alto Palermo, Buenos Aires Design y Patio Bullrich en la Ciudad de Buenos Aires y el Alto Avellaneda en el Conurbano. También la sociedad controladora del Mendoza Plaza Shopping inauguró el Bahía Blanca Shopping, por lo que IRSA tendrá allí también una participación, aunque minoritaria.

59 En el barrio de Caballito (en Buenos Aires), en Neuquén (Provincia de Neuquén) y 20 hectáreas en Rosario (Provincia de Santa $\mathrm{Fe}$ ).

revista invi № 84 / Agosto 2015 / Volumen № 30: 151-177 165 
- IRSA Int. Ltd poseía el 50\% de las acciones y capacidad de votos.

- Parque Arauco -la mayor empresa chilena de administración de shoppings- poseía el 34\%

- Fondo Goldman Sachs de Mercados Emergentes (GSEM/AP Holding LP) administrado por Goldman Sachs y con sede en Islas Caimán era propietario del $8 \%$.

- Accionistas minoritarios -entre ellos, cinco Administradoras de Fondos de Jubilación y Pensión privadas argentinas- se repartían el restante $8 \%$ de las acciones.

Mediante este financiamiento, los empresarios de IRSA buscaron convertir a la empresa "en la principal protagonista de la industria de los centros comerciales en la Argentina" ${ }^{\prime 6}$. Para ellos, el potencial de este segmento tenía que ver principalmente con cambios en los modos y capacidad de consumo de los argentinos, atribuyendo el éxito a:

"(i) el creciente poder adquisitivo de los consumidores; (ii) el cambio en el estilo de compra, abandonando las compras en negocios a la calle, y (iii) el relativamente bajo nivel de penetración en el mercado de los centros comerciales en la Argentina, comparado con muchos países desarrollados, aporta un gran potencial de crecimiento a largo plazo." ${ }^{.11}$

60 IRSA, 1999.

61 Ibid.
Por ello, su política de compras consistió básicamente en comprar los mayores centros comerciales de la ciudad, tanto en volumen de ventas como en cantidad de comercios. Dichos centros se orientaron a todos los sectores sociales, ubicándose en zonas de alta, media y baja renta ${ }^{62}$.

Explotar dicho potencial implicó una especialización en el rubro con la que no contaron hasta 1998. Dicha especialización, plasmada también en la reforma societaria en el segmento de los shoppings, se tradujo en un aumento extraordinario de la tasa de rentabilidad. La misma se explicó durante el período previo a la crisis argentina de 2001 sobre todo por la presión ejercida sobre los inquilinos de los locales comerciales ya que, además del alquiler, APSA cobraba porcentajes que variaban entre el 4 y el $8 \%$ sobre las ventas brutas, un porcentaje para un fondo de promoción, ciertos cargos de administración de ese fondo de promoción, entre otros ítems. De esta manera, la "mejora en los márgenes operativos" de la que hablaban los balances involucró en la práctica un importante aumento del valor de los alquileres, como denunciaban los comerciantes y reflejaban los periódicos de la época ${ }^{63}$.

Para ser más precisos, a junio de 1997 el segmento de los shoppings reportó un resultado operativo de

62 Ver Socoloff, 2013.

63 Soros sube los precios en shoppings, 1998. 
AR $\$ 10.000$ (equivalentes a U\$S 10.000), mientras que a junio de 1998 el resultado se multiplicó exponencialmente, totalizando en sólo un año unos U\$S 11.091.000. Por su parte, para junio de 1999 y de 2000 fue de $\$ 15.598 .000$ y $\$ 12.264 .000$ respectivamente, mostrando en este último caso un leve descenso como preludio de la crisis económica argentina de 2001.

En la Ciudad de Buenos Aires, IRSA realizó entonces las siguientes adquisiciones y desarrollos:

Como se observa en la tabla 2, y como mencionábamos, la mayor parte de las adquisiciones fueron realizadas entre 1996 y 1998, período en el IRSA por medio de APSA se convirtió en el principal administrador de centros comerciales de Buenos Aires.

Importante resulta también destacar que todas las sociedades adquiridas habían sido desarrolladas por diferentes grupos locales o agrupaciones de empresarios, con la sólo excepción del Grupo Pérez Companc (del empresario Gregorio Pérez Companc que figura en Forbes como el tercer empresario más rico de Argentina) ${ }^{64}$ que contaba con participaciones en varios centros comerciales del

64 Gregorio Perez Companc, 2013.
$\mathrm{GBA}^{65}$. Es decir que, con la sola excepción de este último grupo, todos los centros comerciales se originaron como desarrollos llevados a cabo por promotores inmobiliarios locales con apoyo limitado de inversores internacionales.

Mediante estas compras, IRSA adquirió una situación cuasi monopólica en la CABA, comprando agresivamente gracias a la asistencia de socios internacionales y el endeudamiento los más importantes centros comerciales de la ciudad. Se apropió así de segmentos residuales dentro de otros grupos, alcanzando volúmenes de operación inéditos para el segmento. La crisis argentina sin duda complicó los planes de la empresa. Sin embargo, como lo veremos a continuación, el grupo recuperó rápidamente la rentabilidad en todos sus segmentos en sólo tres años.

\section{El "principal negocio" y posición dominante}

Durante la crisis argentina de 2001, la empresa IRSA debió deshacerse de importantes activos y dejó de contar con los aportes del Quantum Fund (Soros). Sufrió además un proceso de restructuración que

65 La venta de las sociedades de los shoppings y de diversas reservas de tierra adquiridas por IRSA se vinculó con la estrategia del Grupo Pérez Companc de deshacerse de la rama inmobiliaria en el marco del proceso de especialización del holding en la explotación agraria y la agroindustria. 
TABLA 2: ADQUISICIONES Y DESARROLLOS DE CENTROS COMERCIALES DE IRSA EN LA CIUDAD DE BUENOS AIRES (1991-2013)

\begin{tabular}{|c|c|c|}
\hline Año & Compras y desarrollos & \\
\hline 1992-1998 & Abasto de Buenos Aires & $\begin{array}{l}\text { Adquirido por la quiebra de la Cooperativa "El Hogar Obrero". Desarrollado } \\
\text { junto con Parque Arauco y Goldam Sachs }\end{array}$ \\
\hline 1996 & Galerias Pacífico & $\begin{array}{l}\text { Adquisición por medio de una licitación pública para la administración del cen- } \\
\text { tro comercial mediante la sociedad "Inversora del Pacífico SA". Luego vendido } \\
\text { al Grupo Sutton (de propiedad del empresario argentino Sutton) }\end{array}$ \\
\hline 1997 & Alto Palermo Shopping & $\begin{array}{l}\text { Adquisición de la sociedad Alto Quilmes SA (de propiedad del empresario } \\
\text { Grosskopf } 25 \% \text { ) y Alto Palermo Shopping Argentino SA (de propiedad del grupo } \\
\text { Pérez Companc) }\end{array}$ \\
\hline 1997 & Paseo Alcorta & $\begin{array}{l}\text { Adquisión del } 100 \% \text { del paquete accionario de New Shopping S.A. (de propie- } \\
\text { dad de las sociedades Inversora Steuer S.A., Vilca S.A. y Otesa S.A. vinculadas } \\
\text { al empresario y político colombiano-argentino Francisco De Narváez) }\end{array}$ \\
\hline 1997 & Buenos Aires Design & $\begin{array}{l}\text { Adquisición como parte del paquete accionario Alto Palermo Shopping Argenti- } \\
\text { no SA (de propiedad del grupo Pérez Companc) y el } 44 \% \text { de la sociedad Empren- } \\
\text { dimientos Recoleta SA (empresa de los empresarios argentinos Trusso) }\end{array}$ \\
\hline 1998 & Patio Bullrich & $\begin{array}{l}\text { Adquisición del paquete accionario en manos de Showcenter S.A. y Bullmaco } \\
\text { S.A. (de propiedad del empresario argentino Nicolás Marccarone) }\end{array}$ \\
\hline $2006-2009$ & Dot Baires Shopping & $\begin{array}{l}\text { Adquirido a las empresas: Phillips y Creaurban SA (Grupo de propiedad del em- } \\
\text { presario ítalo-argentino Macri) y la empresa francesa Auchan y el Grupo San } \\
\text { José (constructora de origen español). Desarrollado por IRSA }\end{array}$ \\
\hline $2007-2013$ & Arcos Gourmet & $\begin{array}{l}\text { Adquisición de Arcos Gourmet SA (de propiedad de varios empresarios locales) } \\
\text { con derecho de explotación de un terreno concesionado por el Organismo Ar- } \\
\text { gentino de Administración de Terrenos Ferroviarios hoy llamada Administración } \\
\text { de Inmuebles Ferroviarios Sociedad del Estado (ADIFISE). Se encuentra en } \\
\text { Desarrollo, pero en conflicto con la justicia. }\end{array}$ \\
\hline
\end{tabular}

Fuente: Elaboración propia en base a datos de las Memorias y Balances IRSA y prensa 
la obligó a reducir drásticamente el personal y achicar la estructura organizativa, fusionando varias empresas del grupo. En este contexto, IRSA no realizó nuevos emprendimientos ni adquisiciones importantes. Asimismo, debió reestructurar su deuda, que había sido tomada en gran proporción en 2001, cayendo en el incumplimiento de pagos.

En ese contexto, y como mencionábamos, IRSA fue progresivamente adquirida por otra empresa del grupo llamada CRESUD, cuya titularidad última se encuentra en sociedades alojadas en paraísos fiscales y no es posible conocer ${ }^{66}$.

Atravesada la mencionada reestructuración, observamos a partir del año 2004 una recuperación acelerada. Fue durante los años que le siguieron, que IRSA con estos nuevos accionistas tomaron control de la subsidiaria APSA a través de la compra de casi la totalidad de las acciones de esta sociedad, efectivizada en 2011, pero observable como tendencia a lo largo de todo el periodo.

Como parte de este proceso, IRSA compró en 2005 las acciones de APSA en posesión del Fondo Goldman Sachs Emergings Markets que fueron repartidas entre los dos socios mayoritarios restantes

66 En todo caso, el fuerte endeudamiento intra-grupo a través de la emisión de obligaciones negociables convertibles en posesión mayoritaria, parece haber servido tanto en el pasado, pero sobre todo en este período, como mecanismo de evasión impositiva y de fuga de capitales, similar al mecanismo de "crédito intrafirma" descripto por Basualdo y Kulfas para otros grupos económicos argentinos. Ver Socoloff. 2013; Basualdo y Kulfas, 2000.
(IRSA y Parque Arauco). Seis años más tarde, en 2011, se adquirieron las acciones en mano del socio chileno Parque Arauco, pasando de esta manera a controlar el 94,9\% de APSA ${ }^{67}$. Así, bajo el control casi total de IRSA, APSA pasó a contar con una participación en trece shoppings distribuidos en varias provincias argentinas (algunos desarrollados por la propia empresa), así como numerosas parcelas para futuros desarrollos. En este sentido, luego de 2004, APSA creció exponencialmente no sólo en activos, sino en extensión geográfica, expandiéndose en varias provincias argentinas.

De modo que, al cierre del ejercicio económico de 2013, APSA tenía una cartera de: seis shoppings en la Ciudad de Buenos Aires, dos en el Gran Buenos Aires, dos en Córdoba, dos en la provincia de Santa Fe, uno en Salta y uno en Mendoza; sumado a dos proyectos recién completados (Arcos Gourmet en la Ciudad de Buenos Aires y otro en la provincia de Neuquén), más un terreno en Caballito (CABA), uno en Paraná (Provincia de Entre Ríos) y uno en San Martín (GBA).

Al respecto, nos preguntamos: ¿por qué los empresarios invirtieron en el segmento de los shoppings?

67 Porcentaje que ascendió aún más en 2012 (95,6\%), producto de la compra de acciones que estaban en manos de tenedores minoritarios. 


\section{FIGURA 3: RESULTADO OPERATIVO SOBRE ACTIVO POR UNIDAD DE NEGOCIO DE IRSA (2001-2012)}

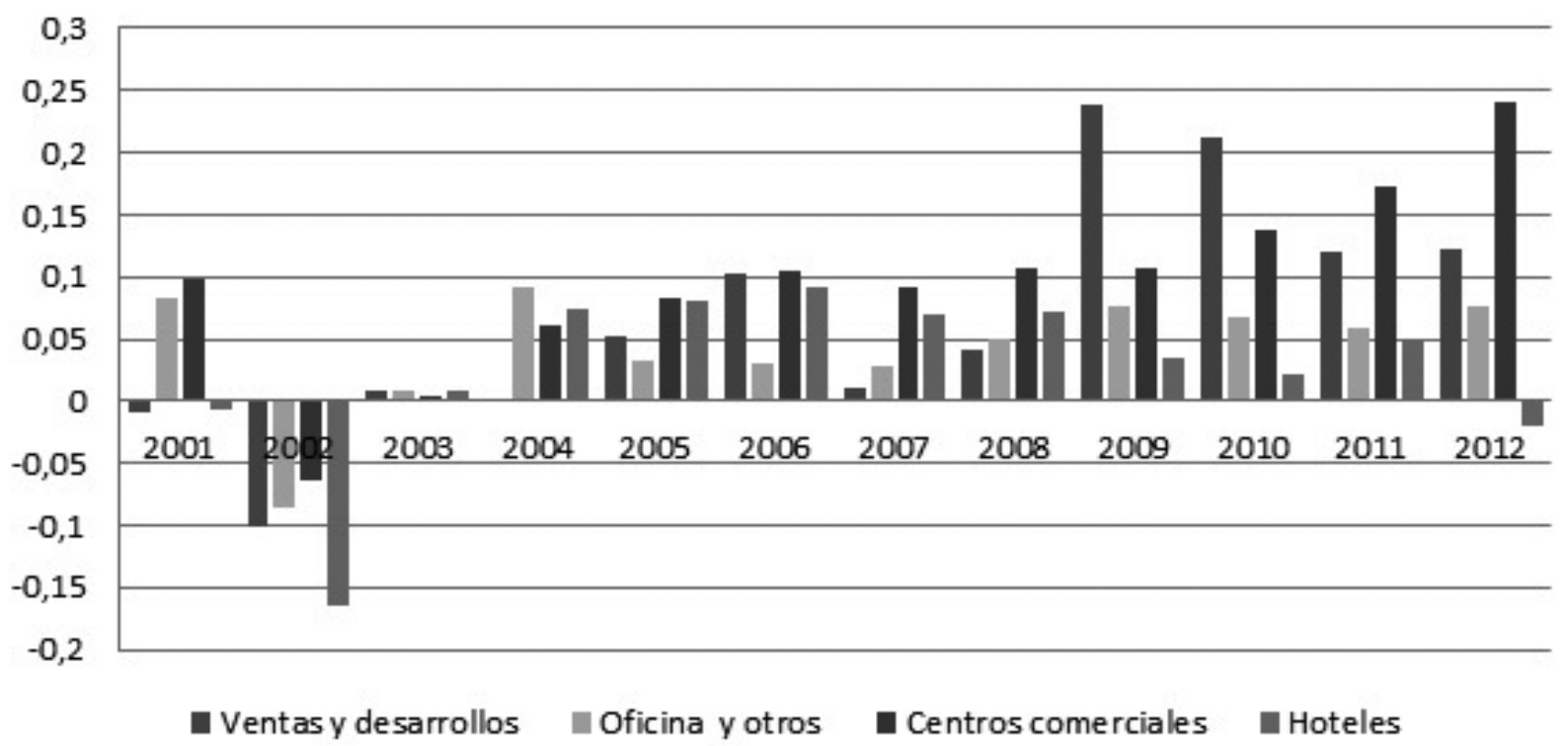

Fuente: Elaboración propia en base a datos de las Memorias y Balances IRSA

Un comienzo de respuesta, a nuestro parecer, tiene que ver sin duda con el crecimiento de la rentabilidad en la postcrisis y con el rápido flujo de caja que ofrece el segmento. Como hemos mencionado ya, el crecimiento del salario real (al menos hasta el año 2008), la expansión del consumo y el crédito a corto plazo, junto con la escasez de otros instrumentos de ahorro, promovieron la orientación al segmento de los centros comerciales.

Todo lo cual, se explica también por el limitado desempeño de los otros segmentos de la compañía (figura 3) como los desarrollos 
residenciales ${ }^{68}$, las oficinas ${ }^{69}$ y los hoteles ${ }^{70}$. El mismo, contrasta con la evolución ascendente para los resultados operativos en el segmento de centros comerciales ${ }^{71}$. En este contexto, la mayor adquisición le va a permitir a IRSA acaparar una porción muy importante del negocio de los shoppings de Argentina, llegando a asumir una posición dominante a nivel nacional (sólo compartida por la cadena Cencosud S.A., única competidora a nivel nacional $)^{72}$, y cuasi monopólica a nivel local, donde APSA no tiene

68 Los cuales sufrieron inestabilidad y fueron orientados al segmento de lujo, producto sobre todo de la falta de financiamiento hipotecario y del crecimiento de los costos de la construcción.

69 Afectadas por la crisis de 2009, con precios en dólares que cayeron para ser recuperados sólo en los últimos años.

70 La devaluación argentina de 2002 tuvo efectos positivos en la ocupación de hoteles, que mostraron mejorías a lo largo de la última década, con excepción de la caída en la tasa de ocupación durante la crisis del 2009 y la epidemia de "gripe A" que azotó Buenos Aires y afectó el turismo local.

71 Cabe aclarar que si bien la tendencia es clara, estos resultados deben ser ligeramente matizados puesto que no existe en los balances un ajuste por inflación de los ingresos, ya que los mismos debían ser expresados en moneda nominal desde el 2003 en adelante. Tampoco existe revaluación de los activos, que son valuados al precio pagado al momento de la compra.

72 Propiedad de Cencosud: Portal de Palermo (CABA), Factory Parque Brown, Factory Quilmes, Factory San Martín, Las Palmas del Pilar Shopping, Plaza Oeste Shopping, Portal Canning, Portal de Escobar, Portal Lomas y Unicenter (GBA), Portal de los Andes (Mendoza), Portal de la Patagonia (Neuquén), Portal de Rosario (Santa Fe), Portal de Tucumán (Tucumán), Portal de Trelew y Portal de Madryn (Chubut). competencia, entre otras razones por la limitada disponibilidad de terrenos ${ }^{73}$.

Con una posición dominante y crecimiento de la rentabilidad de los shoppings, ni siquiera la crisis económica mundial afectó al segmento, a diferencia de lo que ocurrió con los otros rubros. De modo que, aún en plena crisis de 2009, el segmento era el más sólido generador de fondos en Argentina ${ }^{74}$. Todo lo cual va a orientar en los últimos años la estrategia de IRSA definitivamente hacia la actividad de la locación comercial en shoppings, hasta convertirlo en el "principal negocio"75.

73 Como lo explican los documentos de la empresa: "Estamos convencidos que debido a la limitada disponibilidad de grandes terrenos y a las restricciones inmobiliarias en la Ciudad de Buenos Aires, no va a ser sencillo para cualquier compañía competir con nosotros por áreas de desarrollo para centros comerciales." Ver IRSA, 2006.

74 "El negocio de centros comerciales constituye en la actualidad la fuente de generación de fondos más sólida de nuestra cartera de activos." Ver IRSA, 2009.

75 Como vemos en la siguiente cita: "El segmento de Centros Comerciales es nuestro principal negocio y donde pretendemos seguir creciendo y expandiéndonos más allá de nuestro portafolio actual. Tenemos reservas para futuros de desarrollos en la Ciudad de Paraná, en la Ciudad de Tucumán y en el barrio de Caballito en la Ciudad de Buenos Aires". Ver: Memoria y Balance, 2011, p. 5.

revista invi № 84 / Agosto 2015 / Volumen № 30: 151-177 171 
Nuevamente, dicha reorientación se verá facilitada por el ingreso de nuevos accionistas y el reemplazo de los viejos socios internacionales. IRSA focalizará entonces sus inversiones inmobiliarias en Argentina principalmente en este segmento, donde cierto vacío legal en la regulación de los centros comerciales le permite hacerse de altas y estables tasas de rentabilidad.

\section{Conclusiones}

En este artículo nos hemos posicionado entre dos corrientes analíticas que han abordado a los centros comerciales (los micro y los macro análisis) con el objetivo de iluminar a los actores y los procesos que median entre el llamado "capital global" y el "ámbito local". Desde nuestra perspectiva, es gracias a esta mediación que la fijación de capitales se hace posible, remitiendo ganancias a los inversores y transformando el territorio local.

Dicha mediación implica actuar en segmentos determinados del sector inmobiliario en una geografía específica, cumpliendo a la vez las exigencias de rentabilidad de los inversores internacionales. Por ello, nuestro foco estuvo en analizar las estrategias adoptadas por el mayor holding inmobiliario de Argentina (Grupo IRSA) para actuar de canal de inversiones en el sector inmobiliario de capitales internacionales, así como las dinámicas del subsector de centros comerciales (o retail) en Buenos Aires.
La primera constatación es que la orientación hacia los shoppings supo dar cuenta de transformaciones ocurridas en las formas de consumo de la sociedad de Buenos Aires, contribuyendo a la vez a reforzar esas tendencias. Ya que, aun cuando los malls en Argentina poseen una penetración menor que en otros países de la región (como México y Brasil), y compiten con formas de consumo minorista informal o "a cielo abierto", ocupan un lugar privilegiado en la oferta comercial y se han instalado dentro de numerosos grupos sociales como ámbitos seguros de entretenimiento y consumo.

Por otra parte constatamos que la estrategia de IRSA se inserta en el marco de otras estrategias empresariales, produciendo una verdadera transformación en la organización del sector. Y esto porque, si bien la historia del segmento de los shoppings muestra que estos nacieron de la mano de diversos desarrolladores locales como parte de estrategias de grupos económicos diversificados; la especialización de estos grupos -también sometidos a las lógicas de especialización del mercado internacional- condujo a que se deshicieran de estos activos. Lo que observamos entonces fue un proceso de concentración en este sector en manos de agentes especializados en el negocio inmobiliario.

En tercer lugar, advertimos también que por el volumen de capitales involucrados, esta concentración no habría sido posible sin el aporte de grandes capitales financieros internacionales que 
se orientaron a inversiones "alternativas" en países emergentes. Del lado de los inversores, la estructura institucional de IRSA y su participación en el mercado de capitales argentino, les permitió operar en sobre estos activos. Todo lo cual, contribuyó a diversificar sus inversiones tanto en términos tanto geográficos como en tipología de inversión. $\mathrm{Y}$ a pesar de que sabemos que para estos fondos internacionales las sumas destinadas al real estate en las ciudades del hemisferio sur constituyen valores poco relevantes, son estos montos los que han resultado transformadores para las ciudades, como hemos visto en el caso de Buenos Aires.

Por su parte, explicamos también que la orientación de IRSA hacia los activos de renta respondió a estrategias propias de la empresa, que debió lidiar con los condicionantes locales en cada área geográfica y en cada momento histórico. En lo que atañe específicamente a los centros comerciales, IRSA adquirió de manera progresiva una posición cuasi monopólica en la Ciudad de Buenos Aires. Todo lo cual le ha permitido especializarse en el "gerenciamiento" de estos centros, fortaleciendo su capacidad de imponer condiciones a los inquilinos. Al respecto, un detalle que no puede soslayarse es que los mencionados inquilinos se encuentran más fragmentados y dispersos a la hora de negociar, en tanto provienen mayoritariamente del sector textil, que constituye un sector económicamente menos concentrado. A su vez, para estas empresas, la presencia en los centros comerciales resulta imprescindible en el desarrollo de su actividad, debido sobre todo a la importancia de estos espacios en un contexto de transformación en los modos de consumo de los sectores medios.

Sin duda, estos elementos -juntos con otros mencionados aquí- le han permitido a IRSA aumentar la rentabilidad del segmento y consolidarse como la principal empresa de centros comerciales de Buenos Aires, donde el suelo para este tipo de emprendimientos se ha prácticamente agotado, como lo reconocen los empresarios. Su orientación al sector, ha convertido a los shopping centers en su "principal negocio" en la región, distinguiéndose de aquella empresa recicladora de oficinas o desarrolladora de vivienda para sectores medios que caracterizó sus primeros años.

En este marco, concluimos que los administradores locales han sabido actuar como mediadores entre el mundo homogéneo y calculador de las finanzas y la dispersión riesgosa del real estate. Fueron los directivos de IRSA quienes tomaron decisiones de localización, aseguraron pagos regulares de los inquilinos, tasas de rentabilidad elevadas a costa de las cadenas de indumentaria, o aprobaciones de normativas que permitieron desenvolver las actividades de los shopping centers. De modo tal que su mediación activa entre lo global y lo local fue imprescindible para la concentración y consolidación del sector de los shoppings centers, así como para la obtención de tasas de rentabilidad y dividendos por parte de los socios internacionales. 
El devenir de estas relaciones está sin duda ligado a factores no controlados por los directivos de IRSA y a condiciones a las que procurarán adaptarse. Al respecto, cabría preguntarse ¿cómo transformarán la estrategia empresarial las modificaciones realizadas en el funcionamiento del mercado de capitales argentino o la ampliación reciente de los créditos hipotecarios para los sectores medios? ¿Cómo afectarán las transformaciones en los modos y estrategias de consumo de los habitantes de Buenos Aires la dinámica de los shoppings?

Serán seguramente estos los desafíos y los cálculos sociales con los que deberán enfrentarse los empresarios de IRSA no contemplados en los análisis de riesgo homogenizadores de las calificadoras y consultoras internacionales. Es en su rol mediador, cadena de trasmisión de cálculos estandarizados y conflictos locales particulares, donde se juega el devenir de la llamada "globalización" de las ciudades.

\section{Bibliografía}

AMENDOLA, Giandomenico. La ciudad postmoderna: magia y miedo de la metrópolis contemporánea. Madrid, Celeste Ediciones. 2000. ISBN 9788482112404.

AUGÉ, Marc. Los no lugares, espacios del anonimato: una antropología de la sobremodernidad. Barcelona, Gedisa. 1998. 125 p. ISBN 9788474324594.
BASUALDO, Eduardo y KULFAS, Matías. Fuga de capitales y endeudamiento externo en la Argentina. [En línea]. Realidad económica. (173): 76-102, julio 2000. ISSN 0325-1926. Disponible en: http://www.iade.org.ar/modules/noticias/article. php?storyid $=670$.

CAPRÓN, Guénola. Ressemblement et dispersion dans la ville latino-americaine: un nouvel espece public urbain, le cas du centro commercial. Cahiers des Ameriques Latines. 3(35): 21-40, 2000. ISSN 1141-7161.

--- Limites et défis de la gouvernance urbaine en Amérique latine: une analyse à partir de la théorie du régime urbain et de l'opération commerciale d'Alto Palermo à Buenos Aires. [En línea]. Revue de géographie de Lyon. 74 (4): 325-334, 1999. [Fecha de consulta: 30 septiembre 2013] Disponible en: http://www. persee.fr/web/revues/home/prescript/article/ geoca_0035-113x_1999_num_74_4_4982.

--- Les centres commerciaux à Buenos Aires. Annales de la Recherche Urbaine. (78): 55-63, 1998. ISSN 0180-930X.

--- Urbanidad y modernización del comercio: Un análisis a partir del caso de los shopping centers en Buenos Aires. [En línea]. Observatorio Geográfico. 1997. [Fecha de consulta: 30 septiembre 2013]. Disponible en: http://www.observatoriogeograficoamericalatina.org.mx/egal6/Geografiasocioeconomica/Geografiaeconomica/756.pdf.

CARMAN, María. Las trampas de la cultura: los "intrusos" y los nuevos usos del barrio de Gardel. Buenos Aires, Paidós. 2006. 272 p. ISBN 9501265595. 
CATTANEO PINEDA, Rodrigo. Los fondos de inversión inmobiliaria y la producción privada de vivienda en Santiago de Chile: ¿Un nuevo paso hacia la financiarización de la ciudad? [En línea]. EURE. 37(112): 5-22, 2011 [Fecha de consulta: 30 septiembre de 2013]. ISSN 02507161. Disponible en: http://dx.doi.org/10.4067/ S0250-71612011000300001.

CEFID-AR. Informe mensual de préstamos al sector privado no financiero (SPNF) y otros indicadores económicos y monetarios. [En línea]. CEFID-AR. 9(104), diciembre 2012. [Fecha de consulta: 30 septiembre 2013]. Disponible en: http://www. cefid-ar.org.ar/documentos/CEFID-AR-Informe_104.pdf.

CENTNER, Ryan. Microcitizenships: fractious forms of urban belonging after Argentine neoliberalism. [En línea] International Journal of Urban and Regional Research. 36(2): 336-362, 2012. ISSN 0309-1317. Disponible en: http://dx.doi. org/10.1111/j.1468-2427.2011.01050.x.

CICCOLELLA, Pablo. Globalización y dualización en la Región Metropolitana de Buenos Aires: Grandes inversiones y reestructuración socioterritorial en los años noventa. [En línea]. EURE. 25(76): 5-27, 1999. [Fecha de consulta: 30 septiembre 2013]. ISSN 0250-7161. Disponible en: http://dx.doi. org/10.4067/S0250-71611999007600001.

CORPATAUX, José; CREVOISIER, Olivier y THEURILLAT, Thierry. The expansion of the finance industry and its impact on the economy: a territorial approach based on Swiss pension funds. Economic Geography. 85(3): 313-334, 2009. ISSN 1944-8287. Disponible en: http://dx.doi. org/10.1111/j.1944-8287.2009.01035.x.

DAVID, Louise. La production urbaine de Mexico: entre financiarisation et construction territoriale. Une analyse de l'insertion du réseau financier transnational dans les marchés d'immobilier d'entreprise. These de doctorat de l'Universite Paris-Est, 2013.

DAVID, Louise y HALBERT, Ludovic. Logiques financières globales et fabrique de la ville. En: JACQUET, Pierre, ed.; PACHAURI, Rajendra K., ed. y TUBIANA, Laurence, ed. Regards sur la terre 2010. L'annuel du développement durable: Villes changer de trajectoire. París, Presses de Sciences Po. 2010. ISBN 9782724611403.

GORELIK, Adrián. Miradas sobre Buenos Aires: historia cultural y crítica urbana. Siglo Veintiuno Editores, Buenos Aires. 2004. ISBN 9871105932.

GREgORIO Perez Companc. [En línea]. Forbes. 2013. Disponible en: http://www.forbes.com/profile/ gregorio-perez-companc/.

HALBERT, Ludovic. Les acteurs des marchés financiers font-ils la ville ? Vers un agenda de recherche. [En línea] EspacesTemps.net Revue indisciplinaire de sciences sociales. 2013 [Fecha de consulta: 30 septiembre 2013]. Disponible en: http://www.espacestemps.net/en/articles/les-acteurs-des-marches-financiers-font-ils-la-ville-vers-un-agendade-recherche/

revista invi № 84 / Agosto 2015 / Volumen No 30: 151-177 175 
HARVEY, David. The urban roots of financial crises: reclaiming the city for anti-capitalist struggle. En: PANITCH, Leo, ed.; ALBO, Greg, ed. y VIVEK Chibber, ed. The crisis and the left crisis. Socialist register 2012. New York, Monthly Review Press. 2011. ISBN 9781583672532.

--- The enigma of capital and the crises of capitalism. Oxford [England], Oxford University Press. 2010. ISBN 9780199758715.

INSTITUTO Nacional de Estadística y Censos INDEC. Encuesta mensual de shopping centers. 1997 a 2013.

IRSA. Memoria y balance de los años 1989 a 2013.

KOZAK, Daniel. Urban fragmentation in Buenos Aires: The case of Abasto. Thesis Ph.D. Oxford Brookes University, Oxford. 2008.

L.J. RAMOS Brokers Inmobiliarios. Desde los shoppings a las "saladitas". Las distintas modalidades de consumo. Informe del Mercado Inmobiliario. Mayo 2011.

MATTOS, Carlos de. Santiago de Chile, globalización y expansión metropolitana: lo que existía sigue existiendo. [En línea]. EURE. 25(76): 29-56, 1999 [Fecha de consulta: 30 septiembre 2013]. ISSN 0250-7161. Disponible en: http://dx.doi. org/10.4067/S0250-71611999007600002.

NAPPI-CHOULET, Ingrid. Les mutations del'immobilier de la finance au développement durable. Paris, Autrement. 2009. ISBN 9782746713420.

PANIGO, Demián y NEFFA, Julio César. El mercado de trabajo argentino en el nuevo modelo de desarrollo. [En línea] Argentina, Ministerio de Economía y Finanzas Públicas. 2009. [Fecha de consulta: 30 septiembre 2013]. Disponible en: http://www.mecon.gov.ar/peconomica/basehome/panigo_neffa_2009.pdf.

PEREZ, Miguel; SALCEDO, Rodrigo y CÁCERES, Gonzalo. Apropiación y control social en un centro comercial de Santiago: prácticas socioespaciales y significaciones adolescentes. [En línea]. EURE. 38(113): 53-75, 2012. ISSN 0250-7161. [Fecha de consulta: 30 septiembre 2013]. Disponible en: http://dx.doi.org/10.4067/ S0250-71612012000100003.

ROTBART, Demián. Los shoppings de Buenos Aires Transformaciones urbanas y construcción de consumidores. [En línea]. Café de las Ciudades. 9(98), diciembre 2010. [Fecha de consulta: 30 septiembre 2013]. ISSN 2346-9080. Disponible en: http://www.cafedelasciudades.com.ar/urbanidad\%20y\%20economia_98.htm.

SANFELICI, Daniel. Financeirização e a produção do espaço urbano no Brasil: uma contribuição ao debate. [En línea]. EURE. 39(118): 27-46, 2013. ISSN 0717-6236. [Fecha de consulta: 30 septiembre 2013]. Disponible en: http://dx.doi.org/10.4067/ S0250-71612013000300002.

SOCOLOFF, Ivana. Reflexiones en torno a las relaciones entre empresa, estado y ciudad: Un estudio a partir del caso IRSA en Buenos Aires (19912012). Tesis Doctor en Ciencias Sociales, Facultad de Ciencias Sociales, Universidad de Buenos Aires. 2013 
SOROS sube los precios en shoppings. Diario La Nación, Argentina, 26 de febrero de 1998. Disponible online: http://www.lanacion.com.ar/88935soros-sube-los-precios-en-shoppings. (En sección: Comunidad de negocios).

SOROS, George. Soros on Soros: staying ahead of the curve. J. Wiley, Nueva York, 1995. ISBN 978-0-471-11977-7.

THEURILLAT, Thierry. La ville négociée: entre financiarisation et durabilité. [En línea]. Géographie, Economie et Société. 13(3): 225-254, 2011. ISSN 1295-926X. Disponible en: http://dx.doi. org/10.3166/ges.13.225-254 